\title{
Evolução das lesões precursoras do câncer de colo uterino e do câncer de mama em uma cidade no interior do estado do Rio de Janeiro
}

\section{Evolution of precursor lesions of cervical cancer and breast cancer in a city in the interior of the state of Rio de Janeiro}

Gabriela Aparecida Azevedo ${ }^{\dagger *}$, Orlando Antônio Campo DallOrto

Como citar esse artigo. Azevedo, G.A.; Dallorto, O.A.C.; Evolução das lesões precursoras do câncer de colo uterino e do câncer de mama em uma cidade no interio do estado do Rio de Janeiro. Revista de Saúde. 2021 Dez./Mar.; 12 (1): 06-12.

\section{Resumo}

O câncer de mama é uma neoplasia de relevante recorrência e suas taxas de mortalidade também são proporcionalmente elevadas, e, em razão disso, motiva ampla discussão em torno de medidas que promovam seu rastreio. Já o câncer de colo uterino, do mesmo modo, também possui índices de mortalidade ainda elevados e se destaca a necessidade de conscientização das mulheres à realização periódica do exame de colpocitologia oncótica, a fim de detectar precocemente possíveis lesões precursoras de tumores. Realizou-se um estudo transversal, de base populacional na cidade de Vassouras, RJ, no período pré-determinado de 2017 a 2019, a partir das anotações de livro de registro da Ginecologia e revisão de prontuários, perante uma amostra de 130 pacientes para lesões precursoras ao câncer de mama e 70 pacientes para lesões precursoras ao câncer de colo uterino. Foram coletadas informações a partir das pacientes que frequentaram as Unidades Básicas de Saúde do município neste período citado. O objetivo do estudo foi realizar uma análise crítica do seguimento das pacientes ao rastreamento desses cânceres de acordo com os resultados dos exames registrados. A prevalência observada foi de que $76,1 \%$ das pacientes que apresentaram alguma lesão em mamas realizaram o seguimento e $23,8 \%$ delas não o realizaram. Com relação aos dados do câncer de colo uterino, 67,1\% das mulheres que revelaram alguma lesão no colo do útero, mantiveram-se fazendo o seguimento e, 32,8\% delas o abandonaram. Esse trabalho atua como instrumento gerador de mudanças para identificar o grupo de mulheres que não deram seguimento adequado. Dessa forma, tem por finalidade ser um incentivador para o desenvolvimento de futuros estudos que atuem na identificação dos principais fatores do não seguimento, podendo-se obter mudanças em políticas públicas que venham a impactar prognóstico e sobrevida desses subtipos de câncer.

Palavras-chave: Câncer de Mama; Câncer de Colo Uterino; Ginecologia; Saúde da Mulher; Rastreamento.

\begin{abstract}
Breast cancer is a neoplasm of relevant recurrence and its mortality rates are also proportionally high, and, as a result, it motivates a wide discussion around measures that promote its screening. On the other hand, cervical cancer, likewise, also has mortality rates that are still high and the need to raise women's awareness of the periodic examination of oncotic colpocytology is highlighted, in order to detect possible precursor lesions of tumors early. A cross-sectional, populationbased study was carried out in the city of Vassouras, RJ, in the predetermined period from 2017 to 2019 , based on the gynecology record book annotations and medical records review, in a sample of 130 patients for injuries precursors to breast cancer and 70 patients for precursor lesions to cervical cancer. Information was collected from the patients who attended the Basic Health Units in the municipality in the mentioned period. The objective of the study was to conduct a critical analysis of the follow-up of patients to screen for these cancers according to the results of the registered exams. The observed prevalence was that $76.1 \%$ of the patients who had a breast injury underwent follow-up and $23.8 \%$ of them did not. With regard to cervical cancer data, $67.1 \%$ of women who revealed a lesion on the cervix, continued to follow up, and $32.8 \%$ of them abandoned it. This work acts as an instrument that generates changes to identify the group of women who have not followed up properly. Thus, it aims to be an incentive for the development of future studies that work in the identification of the main factors of non-follow-up, and it is possible to obtain changes in public policies that may impact the prognosis and survival of these cancer subtypes.
\end{abstract}

Keywords: Breast cancer; Cervical cancer; Gynecology; Women's health; Screening.

\section{Introdução}

O câncer de mama é o segundo tipo mais frequente entre as mulheres no mundo todo, ficando atrás apenas do câncer de pele do tipo não melanoma․ No Brasil, a distribuição no número de casos incidentes e óbitos por esse câncer apresenta grandes diferenças regionais, com taxas mais altas de acometimento nas regiões Sudeste e Sul e menores taxas nas regiões Norte e Nordeste ${ }^{2-3}$. Segundo a Organização Mundial da Saúde (OMS), cerca de $1 / 3$ dos cânceres podem ser curados se detectados precocemente e tratados adequadamente ${ }^{4}$.

Em relação à distribuição mundial do câncer de mama, curiosamente verificam-se maiores índices do mesmo nas nações desenvolvidas, porém, em contrapartida, também se constata que o maior número de óbitos pelo mesmo não ocorre nesses locais, demonstrando que as políticas de rastreio e de intervenção configuram resultados positivos e são mais bem aceitas e postas em prática ${ }^{5}$. 
Dessa forma, as ações de prevenção ajudam a minimizar o custo de cuidados com a saúde, além de melhorar a qualidade de vida das pessoas. A investigação com detecção dos principais fatores de risco para a doença se constitui em estratégia eficaz para a diminuição do número de casos da mesma.

Ainda não existem métodos totalmente eficazes que possam evitar o surgimento do câncer de mama e do câncer de colo uterino, porém, as medidas de prevenção secundária- $\mathrm{o}$ rastreamento $\mathrm{e}$ a detecção precoce de lesões precursoras de malignidade-, consistem em medidas de extrema importância para minimizar os danos e melhorar o prognóstico da doença em si, permitindo o uso de terapêutica menos agressiva e maior possibilidade de cura.

Alguns fatores têm sido elucidados ao se tentar explicar as causas de não adesão aos métodos de rastreio, sendo os principais associados: renda, escolaridade, idade e a situação conjugal. No câncer de mama, por exemplo, percebeu-se que quanto mais baixo for o nível socioeconômico, pior a adesão a mamografia ${ }^{6}$. Além disso, na percepção de algumas mulheres, os cuidados a saúde estão relacionados apenas a fase reprodutiva.

Ao se tratar do câncer de mama, as estratégias para saúde diferem com respeito ao grupo alvo a ser rastreado, a periodicidade do exame, o número de incidências da mamografia e os exames disponíveis para rastreamento ${ }^{7}$. Em geral, são identificados através desses exames de rastreio, três padrões de lesões: as alterações benignas da mama, lesões malignas in situ e lesões malignas invasivas. Desse modo, a partir de possíveis resultados citados, segue-se a rotina de investigação conforme orientações médicas e diretrizes terapêuticas protocoladas.

Em relação aos dados sobre o câncer de colo uterino, considera-o na posição de terceiro lugar como câncer mais frequente, nesse contexto, as ações de assistência à saúde da mulher e de rastreamento desse câncer tornam-se prioridades para o Sistema Único de Saúde (SUS) e a agenda de pesquisa brasileira. O mesmo é caracterizado pela replicação desordenada do epitélio de revestimento do órgão, comprometendo o estroma e podendo invadir estruturas e órgãos contíguos ou à distância. Existem, para este subtipo, duas categorias de carcinomas invasores dependendo do epitélio de origem acometido: o carcinoma epidermoide que atinge o epitélio escamoso ( $80 \%$ dos casos) e o adenocarcinoma que atinge o epitélio glandular ( $20 \%$ dos casos).

O câncer de colo uterino é uma patologia de desenvolvimento lento, em que mulheres podem permanecer assintomáticas por longo período e já serem portadoras de lesões precursoras, portanto, daí se destaca a relevância das medidas de prevenção secundária.

Sabe-se que devido a elevada magnitude de câncer de colo uterino no contexto em Saúde da Mulher no Brasil, a Organização Mundial da Saúde (OMS) prioriza ações em uma rede organizada para diagnóstico precoce das lesões precursoras e tratamento adequado, já que com o exame de colpocitologia oncótica no período adequado para rastreio, em uma população alvo, é possível reduzir, em média, 60 a $90 \%$ o número de cânceres invasivos deste subtipo na população feminina ${ }^{8}$. Portanto, o câncer de colo uterino também é um exemplo do impacto positivo da detecção precoce na morbimortalidade, conforme verificado em países que organizaram programas efetivos de rastreamento populacional ${ }^{9}$.

Existem fatores já conhecidos que contribuem para a recusa das mulheres em submeter-se ao exame de preventivo, tais como: a desinformação, a dificuldade de acesso às unidades municipais de saúde, proibição de alguns maridos, receito de sentir dor, medo, vergonha e constrangimento ${ }^{10}$. Porém, é preciso ainda verificar posteriormente a existência de outros fatores ainda não conhecidos, que possam, inclusive, constituir-se em características específicas de determinado município e que também atuem como impeditivos ao seguimento adequado dessas mulheres.

Nesse sentido, o presente estudo teve por objetivo realizar uma análise crítica quantitativa do seguimento das pacientes do sexo feminino inseridas nas Unidades Básicas de Saúde (UBS) do município de Vassouras-RJ, cujos exames apresentaram alguma lesão precursora de malignidade e observar o desfecho de tal lesão: se permaneceu inalterada, evoluiu para um estágio acima ou se regrediu. Com esta série de dados, foi possível identificar o número de pacientes no município que se mantiveram em rotina de cuidados recomendada por profissional médico.

\section{Material e Método}

Para a realização do presente estudo transversal, de base populacional na cidade de Vassouras, RJ, no período pré-determinado do mês de janeiro do ano de 2017 ao mês de dezembro do ano de 2019, fez-se uso das anotações de livro de registro da Ginecologia e revisão de prontuários da população feminina adscrita nas 14 Unidades Básicas de Saúde do município de Vassouras, RJ. A pesquisa foi aprovada pelo Comitê de Ética em Pesquisa da Universidade de Vassouras (Parecer $\mathrm{n}^{\mathrm{o}}$ 3.417.083, de 26/06/2019- CAAE 15658919.6.0000.5290) e tendo parecer deferido pelo Núcleo de Educação em Saúde no dia 27/05/2019. A pesquisa científica intitula-se como observacional e quantitativa, com finalidade de verificar pelos números encontrados a continuidade ou não das pacientes aos processos de rastreamento, segundo as condutas recomendadas pelo Ministério da Saúde perante lesões precursoras dos cânceres de mama e de colo uterino.

A partir disso, houve a análise de 200 pacientes, 
sendo 130 exames de mamografia e 70 exames de colpocitologia oncótica, cujos resultados apresentaram algum tipo de alteração, sendo tais mulheres pertencentes às Estratégias de Saúde da Família de Vassouras, totalizando-se 14 unidades. Os dados obtidos foram, ao longo do estudo, sendo tabulados e inseridos em planilha do programa Excel 2016, com exposição dos mesmos ao final do projeto e também com construção de gráficos. O desfecho principal estudado e compartilhado a partir do presente artigo foi o de ter alguma alteração no exame de rastreio para câncer de mama e de colo uterino e verificar se, a partir de tal lesão precursora, houve ou não o seguimento adequado da paciente segundo recomendações médicas preconizadas pelo Ministério da Saúde (MS) e pelo Instituto Nacional de Câncer (INCA) e, como cada uma dessas lesões se comportou ao longo do tempo: evoluiu, regrediu ou permaneceu inalterada.

\section{Resultados}

Foram avaliadas, em totalidade, 200 mulheres adscritas em 14 Unidades Básicas de Saúde do município de Vassouras-RJ, sendo estas, pacientes que apresentaram resultados de exames de mamografia e de colpocitologia oncótica com alguma alteração: carcinoma in situ, cicatriz radial, proliferação epitelial atípica, neoplasias lobulares, lesões papilíferas, lesão fibroepitelial classificadas de acordo com o BIRADS (Breast Imaging Reporting and Data System) ${ }^{11}$ e lesões intraepiteliais escamosas de alto grau na citologia, neoplasias intraepiteliais cervicais 2 e 3 na histologia e adenocarcinoma in situ ${ }^{12}$, respectivamente.

Constatou-se que, no período de dois anos de observação do presente estudo, em relação à mamografia, das 130 pacientes avaliadas, 99 delas, ou seja, $76,1 \%$ deram seguimento ao processo investigativo e de acompanhamento das lesões no tempo desejado, enquanto, 31 delas, ou seja, $23,8 \%$ não deram o prosseguimento conforme thes foi orientado pelo médico ginecologista da UBS conforme: BIRADS 0 - realizar outro exame, como a ultrassonografia de mamas; BIRADS 1 - manter mamografia anual de rotina; BIRADS 2 - manter mamografia anual de rotina; BIRADS 3 -mamografia após seis meses; BIRADS 4 realização de biópsia percutânea; BIRADS 5 -realização de biópsia percutânea; BIRADS 6 - seguimento como câncer ${ }^{13}$ (figura 1).

Ainda em relação aos dados obtidos sobre as lesões precursoras do câncer de mama, dentre as pacientes que mantiveram o rastreio, observou-se que 38 delas ou $38,3 \%$ apresentaram lesões que, ao longo do tempo, permaneceram inalteradas. Além disso, 24 delas ou $24,2 \%$ tiveram suas lesões iniciais regredidas na escala BIRADS, e, finalmente, em 37 do total das mulheres ou 37,3\% delas, a lesão prévia avançou em relação a mesma escala citada (figura 2).

Em relação à epidemiologia do câncer de colo

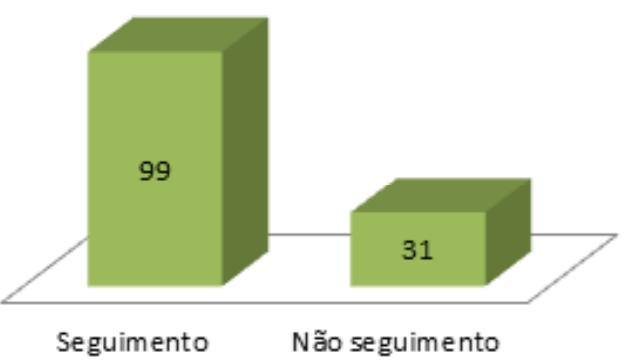

Figura 1. Rastreamento Câncer de Mama no município de Vassouras-RJ.

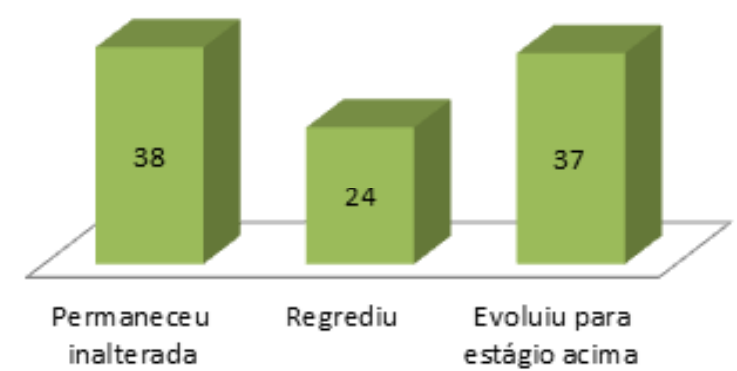

Figura 2. Seguimento Câncer de Mama: o que houve com as lesões?

uterino, a prevalência das condutas de rastreio, para o mesmo período de dois anos, dentre 70 pacientes avaliadas com algum tipo de lesão precursora do mesmo, 47 ou $67,1 \%$ deram o seguimento conforme o que the foi orientado e 23 delas ou $32,8 \%$ não mantiveram a rotina adequada de seguimento conforme os resultados: lesão intraepitelial escamosa de baixo grau ou NIC I - tratar de imediato a lesão ou seguir a paciente por colposcopia ou citologia e posteriormente tratá-la se lesão persistente ou progressiva após 18 a 24 meses; NIC II ou NIC III tratar com CAF ou crioterapia ${ }^{14}$ (figura 3 ).

Dentre as pacientes com alguma alteração na colpocitologia oncótica e que seguiram as orientações do ginecologista, 11 ou $23,4 \%$ delas apresentaram suas lesões inalteradas, enquanto 20 ou $42,5 \%$ tiveram suas lesões iniciais regredidas e, por fim, em 16 delas ou $34 \%$, a lesão avançou (figura 4). 


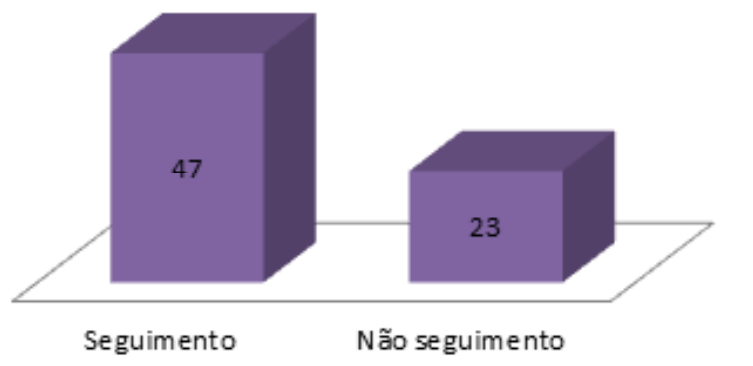

Figura 3. Rastreamento Câncer de Colo Uterino no município de Vassouras-RJ.

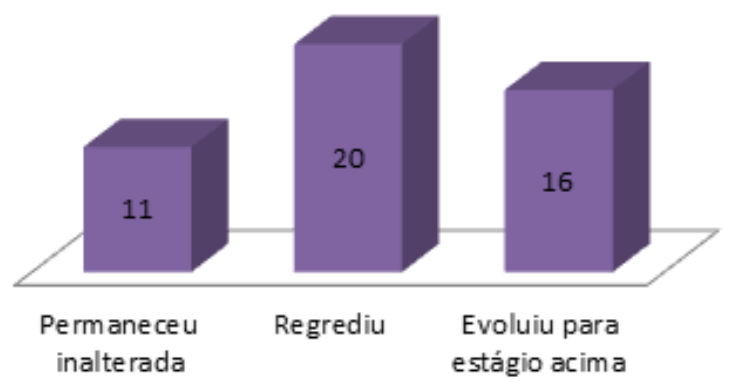

Figura 4. Seguimento Câncer Colo Uterino: o que houve com as lesões?

\section{Discussão}

Ter um diagnóstico situacional de um município é uma ferramenta valiosa que auxilia no conhecimento de problemas e necessidades sociais daquele local, desse modo, permitindo conhecer, por exemplo, como é a adesão da população aos serviços que prezam por diagnóstico precoce e rastreio de câncer. Quando se conhece o perfil de uma cidade e de cada UBS nela inserida pode-se atuar de forma eficaz, visando a resolução das principais questões que ainda fazem com que algumas pacientes não procurem atendimento médico ginecológico de rotina e nem deem a continuidade esperada e aconselhada pelo profissional que as atendeu, sendo esse fato de grande estímulo para a realização do presente estudo e de outras publicações futuras que possam acrescentar algo ${ }^{15}$.

Os parâmetros sociais e de saúde se tornam ainda mais relevantes quando se tratam de questões muito prevalentes na prática médica, como os cânceres de mama e colo uterino. Segundo o INCA, estima-se que, em 2020, sejam contabilizados ainda 66.280 novos casos de câncer de mama e 16.590 de câncer de colo do útero, sendo, portanto, ambos de elevada magnitude e impacto social ${ }^{16}$.

Em relação ao câncer de mama, das 130 pacientes avaliadas no período de dois anos, 76,1\% fizeram o acompanhamento desejado e orientado, evidenciando, dessa forma, que a maioria delas se atentou em manter o rastreamento de suas lesões precursoras, enquanto, uma minoria, 23,9\% não manteve tal preocupação em relação aos seus exames de mamografia. Estudo qualitativo realizado pela Universidade Federal do Rio Grande do Sul em 2009 buscou encontrar possíveis causas para que algumas mulheres ainda não façam seus exames de maneira adequada. Os resultados evidenciaram, em primeiro lugar, o sentimento de vergonha pela exposição corporal, seguidos por dor ou desconforto durante o exame e também pela falta de informações a respeito da doença e seu diagnóstico precoce via TV ou rádio ${ }^{17}$. Tais resultados ressaltam a importância de conteúdo mais acessível para esse público-alvo em suas casas, como a distribuição de cartilhas educativas e explicativas atualizadas, visando maior discernimento sobre questões que já deveriam ter sido superadas.

Para o câncer de colo uterino, sabe-se que a incidência e a mortalidade pela doença podem ser quase que totalmente prevenidas por ações de saúde pública, desde que exista planejamento e a execução das ações sejam corretamente gerenciadas. Das 70 pacientes analisadas, $67,1 \%$ delas realizaram seguimento e $32,8 \%$ não o fizeram. Estudos já realizados concluíram que o principal fator que atua contra o rastreio do câncer de colo de útero é a baixa cobertura, principalmente pela falta de regularidade na coleta do exame de colpocitologia oncótica. Os dados contidos em sistemas de informação do SUS para essa patologia se baseiam em procedimentos e não nas pacientes em si. $\mathrm{O}$ SISCOLO (Sistema de Informação do Câncer do Colo de Útero) registra aqueles casos em que os exames de preventivo realizados no Brasil se mostraram alterados, visando, assim, ter uma estatística geral, porém, a partir disso, não se consegue identificar, individualmente, quais pacientes estão sem controle adequado ou que já realizaram o exame há anos atrás.

Estudo realizado na cidade de Amparo, em São Paulo, mostrou que o rastreamento para o câncer de colo uterino permanece ocorrendo de modo oportunístico ${ }^{18}$, ou seja, apresenta uma tendência gradual ao espaçamento dos controles, assim, as mulheres deixam de realizar o preventivo ao longo dos anos de acordo com a periodicidade recomenda pelo Ministério da Saúde. A distribuição periódica de cartilhas também seria aqui um modo de esclarecer a importância de realizar a colpocitologia oncótica no tempo adequado, mantendo os exames e a saúde em dia.

Com o princípio da territorialização e o cadastramento familiar presente em cada UBS, o objetivo principal é de que os agentes comunitários possam conhecer melhor o perfil de cada pessoa inserida em um domicílio e, deste modo, identifiquem as mulheres que estejam mais expostas aos fatores de risco, justamente por não estarem realizando o rastreamento 
de maneira adequada. Assim, realizar a busca ativa não melhora apenas a cobertura dos programas em saúde, mas, gera maior equidade, assegurando uma relação com as populações mais desfavorecidas e de menor nível socioeconômico, possibilitando um diálogo e uma conscientização mais eficazes.

De acordo com os números encontrados, observam-se indicadores positivos para o município de Vassouras quanto a Saúde da Mulher e às práticas ginecológicas, visto que, conforme o que foi exposto, apesar de não se ter alcançado os $100 \%$ de adesão aos métodos de rastreio, a maioria das pacientes se manteve em seguimento de suas lesões e, ainda, quando comparado aos parâmetros nacionais, segundo a síntese de dados dos sistemas de informação do INCA, a média de mamografias realizadas foi de $60 \%$ no Brasil e $67,9 \%$ na região Sudeste ${ }^{19}$, contrastando com um percentual superior de $76,1 \%$ de mamografias realizadas em Vassouras.

Em relação ao câncer de colo uterino os resultados para o município também se mostraram favoráveis, já que, a média nacional de exames de preventivo realizados foi de $48 \%$ segundo os dados mais recentes do SISCOLO $^{20}$, comparando-se ao percentual de $67,1 \%$ de exames de colpocitologia oncótica realizados em Vassouras, corroborando com a teoria de bons resultados para o município. Tal fato pode ser explicado, de diversas formas, pela existência de profissionais capacitados atuando em ambulatórios, no hospital universitário e nas UBS do município, tendo boa acessibilidade a consultas médicas e aos exames complementares.

Destaca-se ainda a importância da realização de campanhas que descrevam os aspectos clínicos e epidemiológicos do câncer de mama e de colo uterino de maneira mais frequente e clara para as pacientes, inclusive, com a possibilidade de estabelecer através delas, um vínculo entre os acadêmicos da Área de Saúde da Universidade de Vassouras com a Atenção Primária, obtendo um atendimento continuado, com atuação dos mesmos nas campanhas, sob os fatores responsáveis pela não adesão e, depois, nos atendimentos da UBS.

As campanhas seriam ainda uma forma de expor os dados estatísticos que comprovem as quedas nos índices de mortalidade e morbidade por esses cânceres, a partir de práticas devidamente realizadas de rastreio para tais doenças, motivando e atraindo assim aquelas pacientes que ainda não aderiram ao sistema ou que não julgam relevante sua periodicidade e as orientações médicas para acompanhamento de alguma lesão suspeita encontrada, objetivando, assim, ficar cada vez mais próximo da meta: maior adesão possível aos programas que diagnosticam precocemente o câncer de mama e de colo do útero.

\section{Referências bibliográficas}

11. Cavalcante, Fernanda Barbosa, et al. "Prevenção Secundária do Câncer de Mama e redução dos danos gerados pela doença”. 2017; 10 (1): 132.

2. Instituto Nacional de Câncer (INCA). Atlas de mortalidade por câncer. 2015; Disponível em: http:// mortalidade.inca.gov.br/mortalidade. 3. Instituto Nacional de Câncer, José Alencar Gomes da Silva. 2017; Livro ABC do Câncer.

4. Boyle P, Levin B. World cancer report. Lyon: IARC Press; 2008. 5. Instituto Nacional do Câncer (INCA). Incidência de Câncer no Brasil. 2019; Disponível em: http://www.inca.gov.br.

6. Santos A, et al. Fatores Relacionados a não Adesão ao Rastreamento para o Câncer de Mama no Brasil: uma Revisão Integrativa; 2017.

7. Vainio H, Bianchini F. Breast cancer screening. Lyon: IARC Press. 2002; vol. 7

8. World Health Organization. International Agency for Research on Cancer. World Cancer Report. Lyon: IARC; 2008.

9. World Health Organization. National cancer control programmes policies and managerial guidelines. 2 a ed. Geneva: WHO. 2015; p.55-67.

10. Silva ECA, et al. "Conhecimento das mulheres de 18 a 50 anos de idade sobre a importância do exame de papanicolau na prevenção do câncer de colo uterino no município de Turvânia-GO”; 2016.

11. Instituto Nacional do Câncer (INCA). Estimativa da incidência e mortalidade por câncer no Brasil. Brasília (DF): Ministério da Saúde; 2019.

12. Instituto Nacional do Câncer (INCA). Estimativa da incidência e mortalidade por câncer no Brasil. Brasília (DF): Ministério da Saúde; 2019.

13. Nascimento JHRD, Silva VD, Maciel AC. Acurácia dos achados ultrassonográficos do câncer de mama: correlação da classificação BI-RADS e achados histológico. Radiologia Brasileira, v. 42, n. 4; 2009.

14. Alves RR, Teixeira TS, Netto, JC. (2002). Lesões precursoras do câncer de colo uterino. 2018; 14(5), 33-38.

15. Oliveira AME et al. Ações extensionistas voltadas para a prevenção e o tratamento do câncer ginecológico e de mama: relato de experiência. Revista da Escola de Enfermagem da USP. 2012; 46 (1): 240-245.

16. Instituto Nacional do Câncer (INCA). Estimativa da incidência e mortalidade por câncer no Brasil. Brasília (DF): Ministério da Saúde; 2020.

17. Mendonça GDS. Por que as mulheres não realizam o exame de mamografia? Um estudo qualitativo em atenção primária em saúde; 2009.

18. Vale DBAPE et al. Avaliação do rastreamento do câncer do colo do útero na Estratégia Saúde da Família no Município de Amparo, São Paulo, Brasil. 2010; Cadernos de Saúde Pública, 26: 383-390.

19. Oliveira EXG et al. Condicionantes socioeconômicos e geográficos do acesso à mamografia no Brasil. Ciência \& Saúde Coletiva. 2011; v.16: 3649-3664.

20. Dolores MFE et al. Informação do SISCOLO: monitoramento da perda das bases de dados como estratégia de qualificação do sistema. Informação do SISCOLO: monitoramento da perda das bases de dados como estratégia de qualificação do sistema; 2016. 